\title{
Réflexions sur une littérature orale: Les "chants" des anciens Eskimos*
}

\author{
Pierre Léon
}

(Université de Toronto)

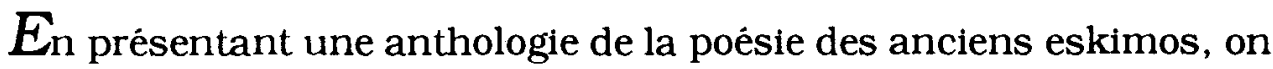
a tenté de mieux faire connaître une étonnante littérature orale, découverte il y a plus d'un demi-siècle par les anthropologues et restée pratiquement inconnue du grand public francophone. L'attention de ce public avait été éveillée par la publication de quelques poèmes du Groënland que Paul-Emile Victor (1959) avait présentés dans une collection qui a disparu. Trente années plus tôt (1929), Madame Thalbitzer avait également traduit des poèmes du Groënland relevés par son mari, l'ethnologue danois. Mais c'est, en France du moins incontestablement à Jean Malaurie (1976) que revient le mérite d'avoir le mieux fait connaître la vie, les coutumes et la philosophie d'un peuple stoïque, trop ignoré de nos modernes contemporains.

Les Chants de la toundra sont un saut en arrière, puisqu'il s'agit là d'un recueil puisé dans le corpus de: Knud Rasmussen (19281945), pour les Iglouliks (vol. 7/1) les Caribous (7/1), les Netsiliks (8/1 et 2), les Cuivres (9); Helen Roberts et Diamond Jenness (1925) pour les Cuivres; et Jean Gabus (1947) pour les Caribous. (J'ai également puisé à d'autres sources moins importantes. Cf. la bibliographie des Chants de la toundra: Ch: 137-139 de mon recueil). Chacun de ces explorateurs apporte ses témoignages et les interprète parfois selon sa personnalité. Il est troublant et touchant à la fois de voir combien dans leur ensemble leurs récits concordent avec ceux, plus récents, de Jean Malaurie. Mais il s'agit là d'une perspective ethnologique que je n'avais pas les moyens d'aborder. Ma préoccupation a été essentiellement littéraire. C'est pourquoi beaucoup de choses, importantes aux yeux des anthropologues, m'auront échappées. J'ajouterai que des raisons éditoriales m'ont contraint à ne garder qu'une centaine des "meilleurs" textes parmi un corpus qui en comporte plusieurs centaines. 
J'étais d'autre part confronté - sauf pour Gabus, qui apporte ses propres traductions françaises - à un problème linguistique. Les textes inuktituts que nous avons ont été traduits en danois ou en anglais, par l'enquêteur lui-même ou parfois plus tard (pour Rasmussen par William Worster et W.E. Calvert, entre 1929 et 1932). Il arrive souvent que la version inuktitute ait posé des interrogations auxquelles on n'a pas encore répondu. Et le côté elliptique des textes, signalé par Jenness, par exemple, vient peut-être autant de problèmes de langue que de culture.

On trouvera donc dans ce volume une sélection très arbitraire, littéraire et subjective dont je demande pardon aux ethnologues. Les textes présentés concernent les seuls Eskimos du Canada (que l'on n'appelait pas encore officiellement Inuit) du début de siècle. Il sont le reflet de la littérature orale des quatre grands groupes représentés - (auxquels j'ai ajouté quelques textes plus récents des Labradors). Ils concernent essentiellement le genre appelé "chant."

Ce chant a toutes les caractèristiques d'un court poème, selon nos normes occidentales; alors que Rasmussen raconte qu'Ivaluardjuk, l'un des grands poètes eskimos, restait ahuri en apprenant l'existence de poètes professionnels chez les Blancs. C'est qu'en fait, tout le monde devait pouvoir creer son propre "chant" chez les Eskimos. Ce chant, on l'improvise à la fin d'un bon repas, d'une veillée, en apprenant une grande nouvelle ou une grande peine. On le récite dans les concours de chants, à la maison des fêtes.

En réalité, il semble qu'il y ait trois catégories de chants. La première serait constituée d'un fonds traditionnel, que toute la communauté se partage, la seconde d'improvisations personnelles et la troisième de poèmes de type professionnel - presque toujours ceux des angakoks, chamanes des groupes. Dans la première catégorie, on trouve d'abord des formulettes fonctionnelles. Ce sont de courts poèmes à réciter dans le besoin: pour guérir d'un mal, pour arrêter une hémorragie, pour avoir des enfants, ou même pour se consoler d'avoir brisé son traîneau:

Je veux rire

Parce que mon traîneau est cassé

Je veux rire 
Parce qu'il s'est cassé les côtes

Je veux rire

Parce que j'ai chaviré

Sur la mauvaise glace.

Ya pas de quoi rire!

(Anonyme, Ch.: 108)

Ce genre de poème fonctionnel est parfois repris par les Angakoks, comme celui-ci - d'un Eskimo netsilik (pour conjurer les blessures):

Toi, comme un pluvier annelé

Toi, comme un canard sauvage

Toi la peau

Pleine de blessures

Pleine de coupures

Va-t'en

Et raccommode-toi!

(Nakasuk, Ch.: 74)

A côté des formulettes fonctionnelles de cette catégorie, on pourrait ranger ėgalement les fables, qui circulent librement. Elles sont à tout le monde. Mais là encore, les angakoks en reprendront un certain nombre à leur compte et leur donneront une forme poétique beaucoup plus élaborée, comme celles du "mariage du hibou d'Atgaralak" ou de "l'ours géant" d'Orpingalik (Chants de la toundra, pp. 25 et 67).

La seconde catégorie est celle du chant personnel. Il ne diffère guère du premier type que parce qu'il appartient en propre à celui qui l'a composé ou troqué, et qu'il n'est pas spécialement fonctionnel. Il présente un intérêt ethnologique parce qu'il décrit surtout les faits de la vie ordinaire, des scènes de chasse, ou tout simplement un animal, tel ce loup, vu d'un joli coup d'oeil impressionniste:

Dans la nuit

Le loup

Devant l'iglou 
Il n'a plus

Que ses deux yeux.

(Anonyme, Ch.: 98)

Ponctué de "Aya-aya," le chant improvisé se termine parfois abruptement par "mon chant est fini, je n'ai plus rien à dire." On peut supposer que le chant personnel s'affine à force d'être récité même si son contenu reste terre à terre, et qu'un jour ou l'autre il passera peut-être dans le domaine public du chant fonctionnel. Ainsi ce chant cité par Gabus, qui décrit des gestes quotidiens et que l'on a pris l'habitude de réciter en jouant au bilboquet, chez les eskimos caribous:

$\begin{array}{ll}\text { Inikpunga } & \text { Je me couche } \\ \text { Inpakpunga } & \text { Je m'éveille } \\ \text { Inerkpunga } & \text { Je fais du feu } \\ \text { Ikipunga } & \text { Je me chauffe } \\ \text { Makitpunga } & \text { Je me lève } \\ \text { Attillipunga } & \text { Je mets mon attilli } \\ \text { Kralikpunga } & \text { Je mets mes pantalons }\end{array}$

Aya - ya

Aya - ya

(Anonyme, Ch.: 15)

Quand on a réussi à composer son chant personnel, on en est propriétaire à tout jamais. Mais si l'on n'a pas d'inspiration, on a recours à l'angakok. C'est de cette manière que naissent une partie des chants constituant la troisième catégorie. L'angakok, en effet, chamane, grand prêtre, guérisseur, doué souvent de qualités physiques exceptionnelles, compose professionnellement ces poèmes. Il les troque contre un bien matériel ou le prêt de la femme de l'acquéreur pour une ou deux nuits. Ce troc lui fait perdre ses "droits d'auteur" mais le poème troqué semble généralement de longueur et de qualité moyenne. Les vrais grands poèmes, l'angakok qui les a composés se les réserve pour les cérémonies publiques où il officie. Même lorsqu'ils sont descriptifs, ces chants ont essentiellement une 
fonction propitiatoire. Il faut se concilier les esprits, les flatter les exalter. D'où une poésie qui va du lyrisme à l'épopée.

Voici le début d'un hymne, de Netsik, grand poète des Eskimos du Cuivre:

Esprit des airs

Viens à moi

Viens à moi

Ton chamane t'appelle...

(Ch. p. 33)

Parfois, l'incantation encadre le poème, qui décrit une scène de chasse, comme dans "Humblement, je suis venu" (Ch.: 42 k'Igpakuhak, eskimo du Cuivre) ou dans le "Chant du phoque" (Ch.: 84) d'Aua, grand poète des Eskimos iglouliks.

On sait que l'angakok récite ce genre de chant lors de cérémonies très théâtrales, que Jean Malaurie (1976) a très bien décrites. On l'accompagne (ou il s'accompagne lui-même) au son du tambour. Cette cérémonie propitiatoire a beaucoup de points communs avec les rites du vaudou. Les ethnologues nous disent que l'angakok peut également improviser de mystérieuses paroles que seuls "ceux qui savent" peuvent comprendre et reprendre. Personne n'en a malheureusement fait de relevés et l'on a avancé l'hypothèse de langues liturgiques anciennes. Il s'agit plus vraisemblablement de glossolalie, langage sans signification, encore très employé dans diverses sectes religieuses, pour communiquer avec les dieux.

Sans aller jusque là, l'angakok peut composer des chants de danse (appelés pisiks par les Cuivres) très hermétiques, pour un occidental. Ainsi cette finale de "l'amoureuse" d'Aguluk ( $\mathrm{Ch}$ pp. 38-39):

J'al continué à marcher vers le sud

Et quand j'ai eu longtemps marché vers le sud,

Longtemps,

Longtemps après,

Je suis arrivé à la Grande Ile. 
L'angakok compose également des chants pour toutes les grandes circonstances de la vie, les bonnes et les mauvaises. Il chante la misère, la faim, le froid, la peur, la famine et la mort. Il chante aussi la joie du chasseur, celle de la ripaille, les fêtes du printemps, les joutes, les concours de chants (poèmes) et de Katajjait (chants de gorge).

Orpingalik, l'un des grands monstres sacrés de la poésie netsilike est, lui, l'auteur de toute une série de chants épiques qui retracent la genèse de l'univers eskimo. Ainsi la création du monde:

La terre était là avant les hommes

Les tout premiers hommes sont sortis

De la terre

De la terre

Tout est sorti de la terre

Même le caribou

Un jour les enfants ont poussé

Hors de la terre

Tout comme les fleurs...

(Ch.: 58)

Les animaux, le soleil, le temps, tous les êtres et les éléments trouvent chez Orpingalik leur explication naturelle dans son système mythologique.

Deux grands thèmes de la poésie universelle sont absents des chants eskimos, celui de l'amour et de la guerre. Comme si, dit John Robert Colombo (1981), les deux passions ètaient intimement liées. On pourrait épiloguer longuement sur le sujet, en partant des récits ethnologiques que l'on possède sur les Eskimos.

On note parmi les poètes un petit nombre de femmes qui ont étè parfois anagakoks. Double transgression puisque les femmes habituellement ne pouvaient pas avoir de chant personnel "parce qu'elles n'avaient pas de mémoire» et elles ne pouvaient pas être chamanes en raison de certains tabous. Voici le début d'un chant de Kibkarjuk (Caribou), femme d'un angakok, qui résume d'un trait la condition féminine eskimo et décrit joliment l'une de ses humbles occupations: 
Je ne suis qu'une femme

Heureuse de mon esclavage

Heureuse de peiner

Avide d'être utile.

Je plume les fleurs de saules

Je plume la mousse et les brindilles

Et voilà que ça me rappelle

La barbe du grand loup ...

(Ch.: 18)

Au plan de la forme, c'est le troisième groupe de chants, qui contient très évidemment le plus haut degré de poésie. Comme dans toute littérature orale la forme du contenu reste simple. On trouve peu d'adjectifs qualificatifs originaux. Le désert est blanc, la mouette grise, l'eau glaciale, etc. A côté de ces stéréotypes, on relève peu de métaphores, mais sous forme de comparaison, d'assez nombreuses et jolies images. Les nuits s'effacent "comme collines dans la brume." "Je sortirai de mon sommeil, preste comme le battement d'aile du corbeau." "Mon esprit se dévidait en néant, comme se déroule la ligne folle".

La forme de l'expression est marquée elle aussi du sceau de l'oralité. Par sa structure linguistique, l'inuktitut repousse à la fin du mot les morphèmes et la rime devient ainsi grammaticale donc facile. On a vu, ci-dessus, l'exemple du "chant du bilboquet." En voici un autre, le début du "chant de la perdrix:"

Utyerpara

Anguwara

Kappiwara

Torkowok

Hiikpok

Hiihanikpok

Tinguiyarpok. Abdguiopok!
Je la guette

Je la chasse

Je lui lance mon javelot, Elle est morte. Je la plume, Ne lui enlève les ailes, Je la coupe en morceaux. Cela me fait de la peine! 
On constate d'autre part, que les auteurs de ces chants ont un bon sens du rythme. Ils y étaient contraints de plus par l'accompagnement au tambour. Dans la récitation, on reprend souvent les mêmes vers, les mêmes refrains et certains poèmes, comme "le chant de la fille qui se changeait en pierre" (Ch. 93) ne comportent qu'un seul couplet que l'on reprend autant de fois que l'on veut, en n'en changeant qu'un seul mot clé chaque fois. Il se crée ainsi une sorte de litanie ou de mélopée, ponctuée de "Aya -ay" répétés inlassablement. De sorte, dit Gabus, qu'un chant de quelques vers peut durer des heures et des heures.

Toute cette récurrence, morphologique, syllabique, paragraphique et lexicale concourt au "brouillage du code" dont parle Jean Cohen (1966), qui est un des aspects les plus évidents de la forme de l'expression poétique. On se drogue du mot répété, dans une ivresse sinon toujours poétique, du moins verbale.

On a donc affaire, avec ce corpus, à une littérature orale très consciente d'elle-même. Et les créateurs de cette poésie ont les mêmes affres, le même orgueil et le même sens du don de l'inspiration que nos modernes poètes. Qu'on en juge par ces courts extraits:

Faire des chansons

Quelle belle occupation

Mais trop souvent

On les rate (...)

Piuviak: "La joie d'un chanteur," (Eskimo netsilik. Ch.: 77);

Ah! mes chansons

Demandent de la force

Et je cherche

Mes mots, oui, moi!

Aua: "Il n'y a que moi que chante,"

(Eskimo netsilik. Ch.: 88);

Les chants sont les pensées

On les chante 
Sur le souffle,

Quand on s'est laissé prendre

Par une grande émotion

Et que la parole ordinaire

Ne suffit plus.

On est pris

Comme un glaçon

A la dérive

Du courant.

Quant les mots dont nous avons besoin

Eclosent d'eux-mêmes

On a un nouveau chant.

Orpingalik: "Les chants et les mots,"

(Eskimo netsilik. Ch.: 57).

Cette brève esquisse typologique des chants eskimos demanderait une étude approfondie de la question par les ethnologues. Elle n'est ici qu'une étape heuristique à propos d'un recueil dont la seule ambition était de sortir de l'ombre une littérature orale trop ignorée. Chaleureuse et efficace, leur poésie est comme le dit un autre grand poète netsilik, Ivaluardjuk, "le sang qui coule du bois le plus sec."

J'ai moi-même êté tenté de chanter ce nord que j'ai beaucoup aimé en découvrant les poètes de mon anthologie. J'ai eu envie d'être des leurs et quelques fragments épars, glanés ça et là, sans suite, ont bourgeonné sous ma plume. En voici quelques extraits. Il sont donc oeuvre commune. Je requière l'indulgence pour la part que j'y ai prise (Voir p. 143.) 


\section{Notes bibliographiques}

Léon, Pierre. Chants de la toundra. Sherbrooke: Naaman et Paris: La Découverte, 1985. L'ouvrage est actuellement distribué par Les Éditions du Soleil, Welland, Ontario.

\section{Autres ouvrages cités}

Cohen, Jean. Structure du langage poétique. Paris: Flammarion, 1966.

Colombo, John Robert. Poems of the Inuit. Toronto: Oberon Press, 1981.

Gabus, Jean. Iglous, vie des Eskimaux-Caribous; mission ethnologique suisse à la baie d'Hudson, 1938-39. Neuchâtel: Victor Attinger, 1947.

Jenness, Diamond. The Life of the Copper Eskimos, Report of the Canadian Arctic Expedition, 1913-18. Vol. 12, Ottawa, 1923.

Mailhot, José. "L'étymologie de "Esquimau" revue et corrigée." Études Inuit, Vol. 2, No 2, 59-69.

Maulaurie, Jean. Les derniers rois de Thulé. Paris: Plon, 1976.

Rasmussen, Knud. Across Arctic America: Narrative of the Fifth Thule Expedition. New York et Londres: G.P. Putman's Sons, 1927. Réimprimé par Greenwood Press, Westport, Conn., 1969.

Rasmussen, Knud. The Fifth Thule Expedition - the Danish Ethnographical Expedition to Arctic North-America, 1921-1924. 10 volumes. Copenhague: Gyldendaloke Boghandel, Nordisk Forlag, 1928-1945. Trad. Angl. W.E. Calvert et N. Worster.

Thalbitzer, William. Légendes et chants esquimaux du Groënland, (région d'Ammassalik), traduits du danois par Mme Hollatz Bretagne. Paris: Ernest Leroux, 1929.

Victor, Paul-Emile. Poèmes eskimos. Paris: Seghers, 1959. 